\title{
Cytosine to Adenosine Transversion Abnormality
}

National Cancer Institute

\section{Source}

National Cancer Institute. Cytosine to Adenosine Transversion Abnormality. NCI

Thesaurus. Code C45650.

A point mutation involving the substitution of Adenosine (a purine base) for Cytosine (a pyrimidine base) in a DNA sequence from eukaryotic or prokaryotic organisms. This abnormality can be either heritable or occur somatically. 